\title{
Divesting Control to the Machine in Digital Art
}

\author{
Fabio Morreale \\ Center for Digital Music \\ Queen Mary University of London, UK \\ f.morreale@qmul.ac.uk
}

\section{INTRODUCTION}

My work explores human-machine collaborations to generate creative outputs. Traditionally, computer agents have been conceived as mere tools to serve the artist. I explore this issue from a different perspective: I aim to research the role of the machine intelligence as a constrain to human control on the creative process. Specifically, I am interested in using forms of machine intelligence to take some of the decisions over the compositional process, leaving the human a limited control on the outcome.

Including constraints in the creative act was exquisitely expressed by Margaret A. Boden (2004):

\footnotetext{
"People often claim that talk of 'rules' and 'constraints'...must be irrelevant to creativity, which is an expression of human freedom. But far from being the antithesis of creativity, constraints on thinking are what make it possible...Constraints map out a territory of structural possibilities which can then be explored, and perhaps transformed to give another one"
}

In this vision, constraining the control on creative activities like music and art is not a ruthless attempt to limit human creativity. On the contrary, divesting some initiative to the machine can produce a set of creative reactions and feelings that are at times similar and at times richer than simply constraining the interaction possibly.

So far, my investigation has been focused on the musical domain. I developed several interfaces and instruments in which the creative activity of music making is limited by autonomous agents. For instance, Chimney (Figure 1) is a software instrument that forces the musician to delegate timing decisions to a non-responsive autonomous agent (Morreale \& Masu 2016). Consequently, the human has limited control on the temporal evolution of the piece.
Another example is Beatfield, a tangible musical interface in which the player can move pieces on an augmented board and control the rhythm of an algorithmically generated piece, whereas timber, harmony and melody are entirely under the control of the algorithm (Masu et al. 2016).

A further example is Robin, a rule-based algorithmic composer that allows users to interact with the composition only to a limited extent (Morreale \& De Angeli 2016). The system automatically generates a classical-like music and the user control is limited to decide the emotional connotation of the piece. The next section extends this reflection to the visual domain.

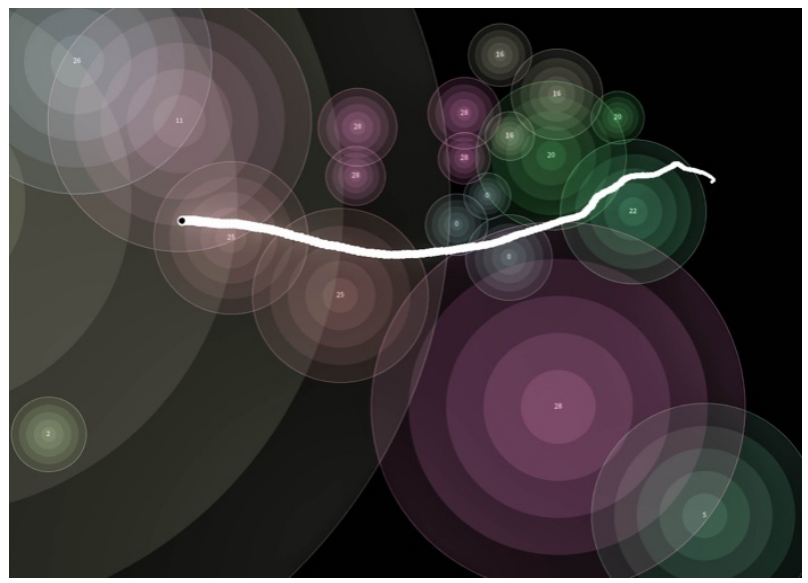

Figure 1:Screenshot of Chimney. The white path is the trace left by the algorithmic walker. The circles are the sonic material added by the human performer.

\section{DRAWING AGENTS}

I recently investigated the concept of divesting control to the machine to produce visual artworks. The paradigm is the same: a more balanced collaboration in which the machine is not a mere tool at my service but an active agent that can take decisions that I cannot contrast.

I developed a system in Processing in which I can control the initial position of a virtual pen on a 
virtual canvas. As I trace a line, several autonomous agents come into existence from the trace and independently start roaming throughout the canvas leaving a trace behind them. The human can then decide the location of the origin of the agents but he is neglected control on their evolution.

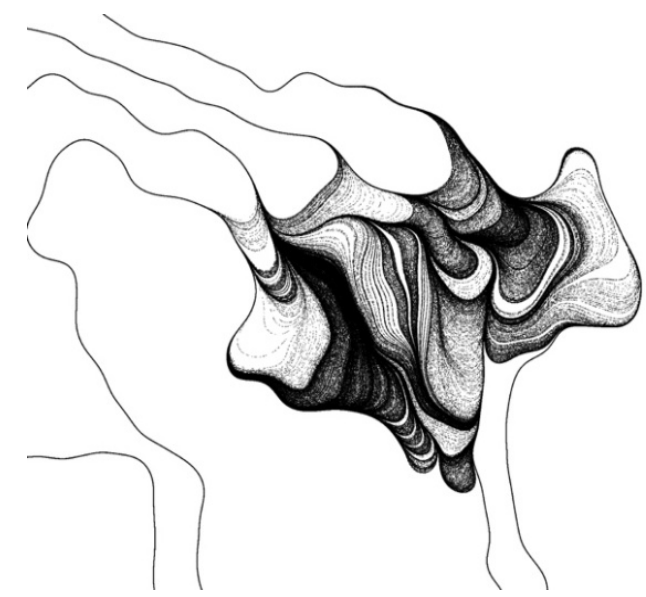

FigureError! Reference source not found.: Image generated with scale $=800$ and strength $=30$

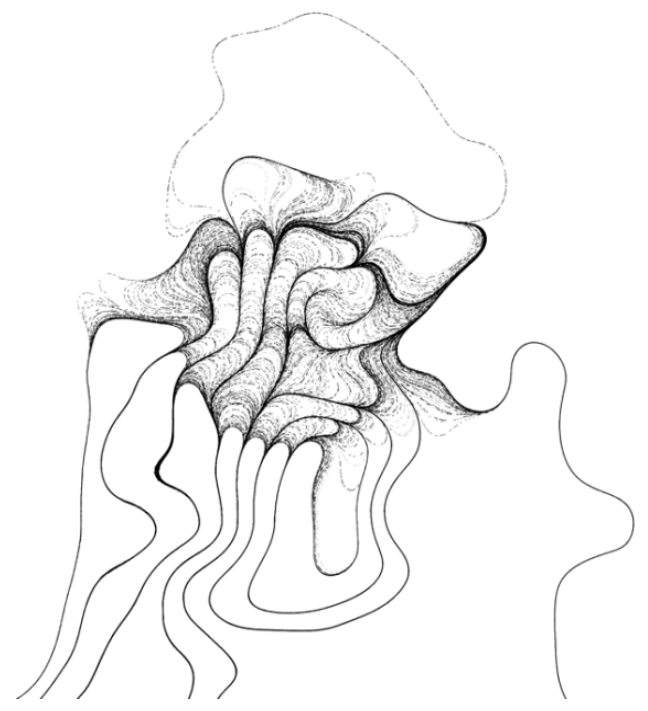

Figure 3: Image generated with scale $=1400$ and strength $=140$

The behaviour of the agents can be modelled on any mathematical function. In the examples shown in Figure 2 and 3, the behaviour of the agents is modelled on an adapted version of the Perlin Noise (Perlin 1985). The Perlin Noise is a random generator function originally developed to produce natural looking textures on computer generated surfaces. This function produces a more natural, harmonic succession of numbers than that of pure random functions.
Add each new frame, each agent computes its next movement as a function of the Perlin noise. Specifically, the direction of the agent at time $I, \alpha_{l}$, is computed as:

$$
\alpha=\text { perlin }\left(\frac{x_{t}}{\sec l e}, \frac{y_{t}}{\text { seale }}\right) \times \text { strength }
$$

where $x_{i}$ and $y_{i}$ are the coordinates of the agent at time $i$; and scale and strength are two constant real numbers used to tune the drawing (see Figure 2 and 3 ). The successive position of the agent is then computed as:

$$
\begin{aligned}
& x_{i+1}=\operatorname{cns} f t * s t a p \\
& y_{t+1}=s \ln \alpha * s t e p
\end{aligned}
$$

where step is a constant real number that determines the length of the movement.

Once the autonomous agents start drawing traces on the screen, the human has no way of intervening with their movements. Despite being constrained by this limitation, the human is not relegated to the role of the spectator but can still be involved in the creation of the artwork by creating new agents.

Divesting part of the control to the machine in digital art thus offers artists the possibility of exploring new possibilities. Without the possibility of entirely influencing the evolution of the piece, the artist is pushed to find creative workarounds and to surrender to a more balanced collaboration with the machine.

\section{REFERENCES}

Boden, M. A. (2004) The Creative Mind: Myths and mechanisms. Psychology Press.

Masu, R., Conci, A., Menestrina, Z., Morreale, F., and De Angeli, A. (2016) Beatfield: An OpenMeaning Audiovisual Exploration. In COOP 2016, pp. 309-314. Springer International Publishing.

Morreale, F. and Masu, R. (2016) Renegotiating Responsibilities in Human-Computer Ensembles. In Proc. Colloquio di Informatica Musicale, Cagliari, Italy, 28 September - 1 October.

Morreale, F., and De Angeli, A. (2016). Collaborating with an Autonomous Agent to Generate Affective Music. Computers in Entertainment (CIE), 14(3), p. 5.

Perlin. K. (1985). An image synthesizer. ACM Siggraph Computer Graphics, 19(3), pp. 287-296. 